\title{
Intraoperatively local infiltration anesthesia in hemiarthroplasty patients reduces the needs of opioids: a randomized, double-blind, placebo-controlled trial with 96 patients in a fast-track hip fracture setting
}

\author{
Janne K HOFSTAD ${ }^{1,2}$, Jomar KLAKSVIK ${ }^{1,2}$, and Tina S WIK ${ }^{1,2}$
}

\author{
1 Orthopaedic Research Centre, St Olavs Hospital Trondheim, Department of Orthopedic Surgery, Trondheim University Hospital, Trondheim Norway; \\ ${ }^{2}$ Norwegian University of Science and Technology, NTNU Trondheim, Norway \\ Correspondence: janne.k.hofstad@ntnu.no \\ Submitted 2021-08-07. Accepted 2021-10-13.
}

Background and purpose - Local infiltration analgesia (LIA) is commonly used as a component in multimodal analgesia. Pain management directed towards hip fracture patients operated on with hemiarthroplasty is often based on knowledge regarding pain treatment following elective surgery. In this elderly patient population, it is of value to clarify whether adding local infiltration analgesia (LIA) to the postoperative analgesic regimen might reduce postoperative pain or have an opioid-reducing effect.

Patients and methods - 96 hip fracture patients undergoing hemiarthroplasty in spinal anesthesia were included. All patients received a multimodal pain regimen and were randomized to receive either ropivacaine or placebo. All patients received morphine depot-opioid and morphine as rescue medication postoperatively. The primary endpoint was pain during mobilization in the recovery unit on the day of surgery. Secondary endpoints were pain during mobilization the day after surgery and postoperative opioid requirements on the first postoperative day.

Results - The levels of pain (NRS) during mobilization both in the recovery unit and on the day after surgery were similar in the 2 groups, with median 4 and 0.5 in the placebo group and median 3.5 and 1 in the ropivacaine group respectively. Total consumption of opioids on day 0 and day 1 were $4.6 \mathrm{mg}$ lower in the ropivacaine group $(\mathrm{p}=0.04)$. Pain during mobilization was registered for only 44 of 96 patients for several reasons, including lack of mobilization.

Interpretation - There were similar pain scores in both the local infiltration and placebo group postoperatively. However, substantially reduced opioid consumption was found in patients receiving LIA.
One of the key parameters to faster recovery after hip fracture surgery is an optimized perioperative pain regimen (1). The management of pain in hip fracture surgery usually involves use of opioid drugs. Opioids are known to cause a wide range of adverse effects such as nausea, somnolence, respiratory depression, and postoperative delirium $(2,3)$. The fragile hip fracture population are at high risk of serious perioperative complications, and opioid use increases this risk (4). Postoperative pain is still the most challenging factor that hinders early mobilization and rehabilitation after hip fracture surgery (5).

Multimodal analgesia for acute postoperative pain is commonly used in elective arthroplasty surgery (6). The concept of the multimodal pain regimen is to combine different analgesics modulating different parts of the pain treatment to reduce the use of opioids and thereby reduce the risk of opioid-related adverse events (7). A multimodal pain regimen following arthroplasty usually consist of spinal anesthesia, opioids, gabapentin, dexamethasone, NSAIDS, acetaminophen, and local infiltration analgesia (LIA) (8). For total knee arthroplasty patients LIA has been found effective (9), whereas the same is not found for total hip arthroplasty patients (10).

There are surprisingly few studies investigating the postoperative pain management following hemiarthroplasty in hip fracture patients $(\mathbf{1 1}, \mathbf{1 2})$. The pain management is often based on knowledge regarding pain treatment of elective total hip arthroplasty patients, although both the nature of the injury and the patient population are quite different. It is therefore important to clarify whether adding LIA to the perioperative analgesic regimen might reduce postoperative pain or the need for opioids for hip fracture patients undergoing hemiarthroplasty. The potential gain of optimized pain treatment and reduction of opioid side effects is high for this vulnerable patient group. 
We investigated the effect of adding perioperative LIA with ropivacaine to a multimodal analgesic regime without NSAIDs, with respect to pain intensity and opioid use in hip fracture patients receiving hemiarthroplasty.

\section{Patients and methods}

\section{Patients}

All patients admitted to the hospital with femoral hip fracture scheduled for hemiarthroplasty were screened for inclusion in this study from December 2015 to May 2018. Patients were included regardless of age and ASA score. Exclusion criteria were patients not competent to give consent to the study, patients operated on with a different surgical approach than the direct lateral approach, patients receiving general anesthesia, and patients receiving femoral nerve blockade less than 4 hours before surgery. Removal of osteosynthesis material in the same surgery also led to exclusion from the study. Finally, patients with contraindications to receiving any part of the multimodal anesthetic regime such as ropivacaine, dexamethasone, acetaminophen, and spinal anesthesia were also excluded from this study.

\section{Randomization and blinding}

Surgeons and healthcare personnel in the post-anesthesia care unit (PACU) as well as the patients were blinded to the type of treatment. Patients were randomized to receive either infiltration with local anesthesia or placebo (saline). The patients were screened for inclusion after admission to the orthopedic trauma unit by the orthopedic resident on call. Sealed numbered envelopes were opened by the operating nurse on the day of surgery. LIA or placebo was prepared in 3 unmarked 50 $\mathrm{mL}$ syringes by the operating room nurse before the patient, the anesthetic personnel, and the surgeon arrived in the operating room.

\section{Study intervention}

All patients were on a standardized clinical fast-track pathway and transported directly to the orthopedic trauma ward unit after their radiographs were obtained to avoid unnecessary waiting time. While still ambulatory the patients were treated with oxygen, fluid, and morphine as needed. As soon as possible after admission to hospital, the patients received a femoral hip blockade consisting of ropivacaine $2 \mathrm{mg} / \mathrm{mL}$ up to $40 \mathrm{~mL}$. Standard preoperative pain treatment was acetaminophen $1 \mathrm{~g}$ every 6th hour and morphine $2.5-5 \mathrm{mg}$ as needed. Preoperatively they were given a single dose of dexamethasone 16-20 $\mathrm{mg}$. A standardized program for intraoperative fluid administration was followed, consisting of Ringer's acetate and antibiotic prophylaxis with cephalothin $2 \mathrm{~g}$ given 30-60 minutes before surgery. After surgery, the patients received Dolcontin $10 \mathrm{mg}$ every 12th hour as depot-opioid and morphine intravenously or orally as rescue opioid (Table 1).
Table 1. Medications throughout the study

\begin{tabular}{|c|c|c|c|c|}
\hline Medication & $\begin{array}{l}\text { Pre- } \\
\text { opera- } \\
\text { tive }\end{array}$ & $\begin{array}{l}\text { Pre- } \\
\text { medi- } \\
\text { cation }\end{array}$ & $\begin{array}{l}\text { Peri- } \\
\text { opera- } \\
\text { tive }\end{array}$ & $\begin{array}{l}\text { Post- } \\
\text { opera- } \\
\text { tive }\end{array}$ \\
\hline Morphine $(2.5-5 \mathrm{~mL}$ iv) & $\mathrm{x}$ & & & \\
\hline Acetaminophen ( $1 \mathrm{~g}$ every $6 \mathrm{~h}$ ) & $\mathrm{x}$ & $\mathrm{x}$ & $\mathrm{x}$ & $\mathrm{x}$ \\
\hline $\begin{array}{l}\text { Femoral hip blockade } \\
\text { ropivacaine } 2 \mathrm{mg} / \mathrm{mL} \text { up to } 40 \mathrm{~mL} \\
>4 \mathrm{~h} \text { before surgery }\end{array}$ & $\mathrm{x}$ & & & \\
\hline Dexamethasone $(16-20 \mathrm{mg})$ & & $\mathrm{x}$ & & \\
\hline $\begin{array}{l}\text { Spinal anesthesia } \\
\text { bupivacaine } 0.25 \% \text { plain } 11 / 2-4 \mathrm{~mL} \\
\text { with added fentanyl or epinephrine }\end{array}$ & & & $\mathrm{x}$ & \\
\hline Cephalothin (2 g i.v.) & & & $\mathrm{x}$ & \\
\hline Morphine depot $10 \mathrm{mg} \times 2$ & & & & $\mathrm{x}$ \\
\hline Rescue opioid i.v./orally & & & & $\mathrm{x}$ \\
\hline $\begin{array}{l}\text { Ropivacaine (saline) } 2 \mathrm{mg} / \mathrm{mL} \\
\text { maximum } 4 \mathrm{mg} / \mathrm{kg}\end{array}$ & & & $\mathrm{x}$ & \\
\hline
\end{tabular}

All patients were scheduled for surgery within 24 hours and operated on between 8 am and $1 \mathrm{pm}$. All patients were operated on under spinal anesthesia with bupivacaine $0.25 \%$ plain $1 \frac{1}{1 / 2}-4 \mathrm{~mL}$ and added fentanyl or epinephrine on an individual basis, preferably at the L2/L3 or the L3/L4 vertebral interspace.

The patients were randomized to receive either LIA or placebo. The LIA consisted of ropivacaine $2 \mathrm{mg} / \mathrm{mL}$ up to $4 \mathrm{mg} /$ $\mathrm{kg}$ with an added $0.5 \mathrm{~mL}$ of epinephrine $1 \mathrm{mg} / \mathrm{mL}$. The placebo group received saline $0.9 \%$. Both groups had a maximum of $150 \mathrm{~mL}$ liquid infiltrated around their hips in a standardized manner after relocation of the hip joint intraoperatively. The first $50 \mathrm{~mL}$ was injected in the periacetabular remaining capsular tissue, and $50 \mathrm{~mL}$ was inserted in the gluteus muscles and the proximal part of the iliotibial tract. The last $50 \mathrm{~mL}$ was inserted in the subcutaneous layers (8).

After surgery, the patients followed a standardized fast-track clinical pathway for hip fracture, and whenever possible they were mobilized in the PACU as soon as the effect of the spinal anesthetic had worn off. Thereafter the patients were transferred to a specialized trauma unit with a well-defined and experienced program for multimodal orthogeriatric rehabilitation (13). On the 1st postoperative day, the patients whenever possible were mobilized with help from a physiotherapist.

\section{Study parameters}

The primary endpoint was pain sensation during mobilization in the PACU (day 0). The secondary endpoint was pain during mobilization in the orthopedic trauma unit on the 1st postoperative day after surgery (day 1), and total opioid consumption on day 0 and day 1 .

Pain at rest and pain during mobilization were registered using the numeric rating scale for pain (NRS), on day 0 and on day 1 . The patients were asked a standardized question: "On a scale from $0-10$, where 0 is no pain and 10 is the worst 


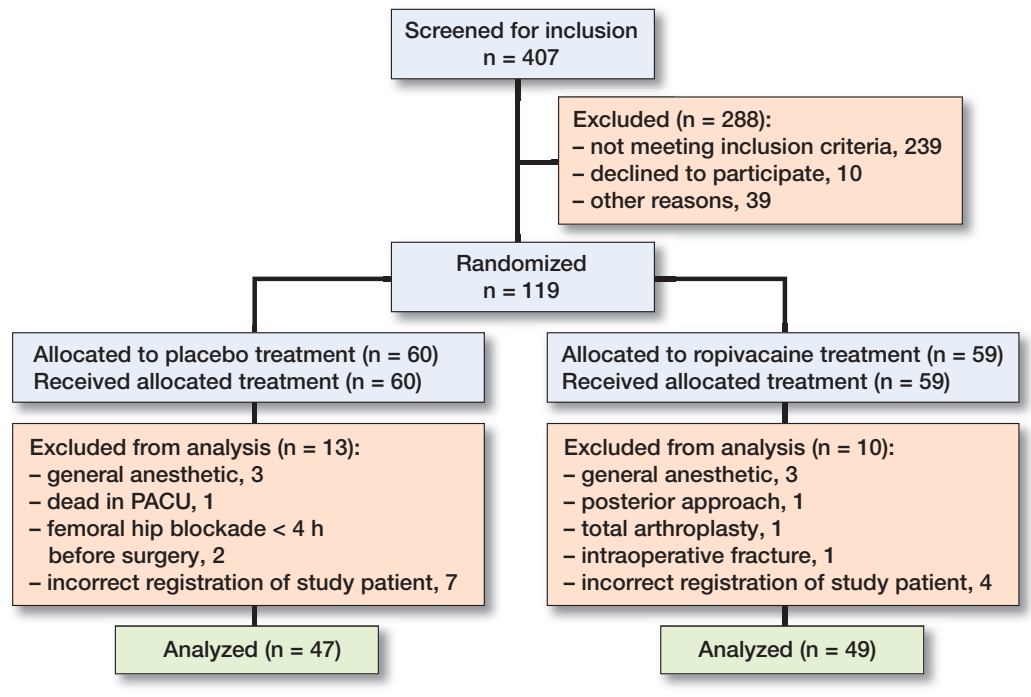

Figure 1. CONSORT 2011 flow chart of patients throughout the study.

imaginable pain, can you define the pain you have right now?"

Mobilization in the PACU was defined as movement of the legs while in bed, sitting on the bedside, or standing next to the bed. All patients were mobilized with a walker in the orthopedic ward unit on day 0 or day 1 depending on the time of surgery. Patients arriving in the ward unit late at night were mobilized on day 1 .

The use of opioids was registered on day 0 and on day 1 . In addition, the length of hospital stay was registered.

\section{Statistics}

With a level of significance of $0.05 \%$, and a power of $80 \%$, a 1-point difference in NRS will be detected with 47 patients in each group. We aimed to include 120 patients in the study to account for dropouts.

The continuous numeric variables for pain score were tested for normality of distribution with histogram and QQ plot. The 2 groups were compared with the Mann-Whitney U-test, as data were not normally distributed. Pain score and total consumption of opioids are presented as median (range). As pain score was collected for only half of the included patients, the median group results are presented descriptively.

Table 2. Baseline demographics

\begin{tabular}{lll}
\hline Factor & $\begin{array}{l}\text { Placebo } \\
(\mathrm{n}=47)\end{array}$ & $\begin{array}{c}\text { Ropivacaine } \\
(\mathrm{n}=49)\end{array}$ \\
\hline Mean age (range) & $83(69-94)$ & $82(68-98)$ \\
Female sex, $\mathrm{n}$ & 32 & 36 \\
Median ASA (range) & $3(1-4)$ & $3(2-4)$ \\
Mean BMI & 23.4 & 23.6 \\
Mean minutes of surgery (range) & $81(55-119)$ & $73(43-139)$ \\
Surgery within 24 h, $\mathrm{n}$ & 44 & 47 \\
Median LOS, days (range) & $5(3-11)$ & $5(3-9)$ \\
\hline
\end{tabular}

Morphine, oxycodone, oxycodone depot, and ketobemidone were calculated using the equianalgesic ratio of each opioid relative to morphine $(14,15)$.

A generalized linear mixed model (GLMM) was used to analyze opioid use on day 0 and day 1. Square-root transformation was applied to the dependent variable to obtain normality of model residuals. Model estimates presented are backtransformed to the original scale. The model consisted of 2 groups and 2 time points as fixed factors, adjusted for age, sex, and ASA. Normality of residuals was verified by histogram.

All statistical analyses were conducted with IBM SPSS version 26 (IBM Corp, Armonk, NY, USA).

\section{Ethics, registration, and potential conflict of interest}

Written and verbal consent was obtained from each patient before inclusion in the study, and the study was conducted in accordance with the Declaration of Helsinki. The project was approved by the regional committees for medical and research ethics (16/1201). The study was registered at ClinicalTrials.gov (identifier NCT02585011). This research did not receive any funding. No competing interest declared.

\section{Results}

407 patients were considered for inclusion, of whom 119 were included and randomized. The most common cause for exclusion was dementia and comorbidity. 47 patients in the placebo group and 49 patients in the ropivacaine group were included in the analyses (Figure 1 and Table 2).

52 of the patients could not indicate pain scores during the first mobilization due to lack of mobilization, fragility, comorbidity, and lack of nursing staff resources. Pain scores were not given for 25 of 47 patients in the placebo group and 27 of 49 patients in the ropivacaine group. Opioid consumption was provided for all patients (Table 3 ).

Table 3. Results. Values are median (range)

\begin{tabular}{lccccc}
\hline & \multicolumn{2}{c}{ Placebo } & \multicolumn{3}{c}{ Ropivacaine } \\
Pain at & $\mathrm{n} \quad(\mathrm{n}=47)$ & $\mathrm{n}$ & $(\mathrm{n}=49)$ & $\mathrm{p}$-value \\
\hline Day 0 & & & & & \\
$\quad$ NRS mobilized & 22 & $4(0-9)$ & 22 & $3.5(0-7)$ & \\
$\quad$ NRS at rest & 30 & $0.5(0-6)$ & 33 & $1(0-8)$ & \\
$\quad$ Opioid use, mg & 46 & $10.3(0-52.5)$ & 49 & $7.5(0-45)$ & 0.2 \\
Day 1 & & & & & \\
$\quad$ NRS mobilized & 22 & $5(3-10)$ & 23 & $5(0-10)$ & \\
$\quad$ NRS at rest & 21 & $0(0-5)$ & 24 & $1(0-6)$ & \\
$\quad$ Opioid use, mg & 47 & $15.0(0-60)$ & 49 & $15.0(0-76.5)$ & 0.5 \\
\hline
\end{tabular}




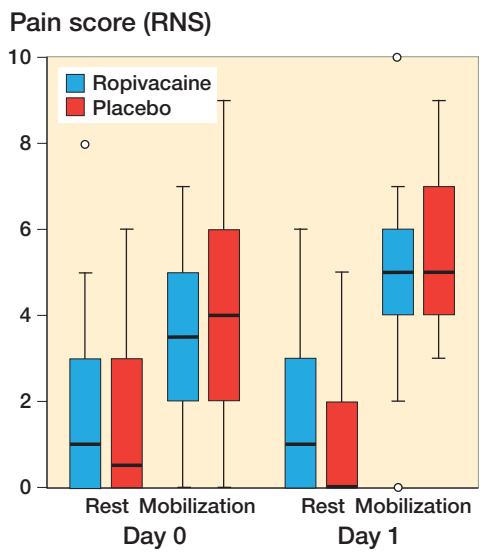

Figure 2. Boxplot showing pain score (NRS) during mobilization on day 1 and on day 0 . The horizontal lines indicate median. The boxes represent interquartile range, and the whiskers extend to the minimum or maximum case/value within 1.5 times height of the boxes.

\section{Primary outcome}

The median pain score during mobilization on day 0 was 4 $(0-9)$ and $3.5(0-7)$ in the placebo and ropivacaine group respectively. The median pain score during mobilization on day 1 was 5 in both groups (Figure 2). 44 and 46 patients were mobilized with a walker on the first postoperative day in the ropivacaine and the placebo group respectively.

\section{Secondary outcome}

Median opioid consumption on day 0 was $10(0-53)$ and 7.5 (0-45) $\mathrm{mg}$ in the placebo and ropivacaine group respectively $(\mathrm{p}=0.2)$, whereas median opioid consumption on day 1 was $15(0-60)$ and $15(0-77) \mathrm{mg}$ in the placebo and ropivacaine group respectively $(\mathrm{p}=0.5)$ (Figure 3 ). Comparing the opioid use between the 2 groups for day 0 and 1, controlling for age, sex, and ASA score, the model estimate for the placebo group was $14.3 \mathrm{mg}$ and $9.7 \mathrm{mg}$ for the ropivacaine group $(\mathrm{p}=0.04)$.

\section{Discussion}

This study aimed to evaluate the effect of intraoperative LIA given to hemiarthroplasty patients on pain intensity and consumption of opioids on the first postoperative day. Pain scores were similar between the groups, but registered for only approximately half the patients and therefore not tested statistically. The median opioid consumption was substantially less in the LIA group when accounting for the covariates age, sex, and ASA group, indicating some effect of intraoperative LIA for hemiarthroplasty patients.

Our findings of reduced opioid consumption are in accordance with 2 other randomized trials on hemiarthroplasty patients $(\mathbf{1 1}, \mathbf{1 2})$. These studies also found reduced pain for the LIA group. There are some differences in the studies that might explain this: in one of the studies NSAIDs were added to the LIA (11). In addition, the LIA group received preemptive analgesic medication whereas the control group did not. Both adding NSAIDs and opioids to the LIA compound and giving preoperative opioids to only one group complicates the identification of which component contributed to the outcome, making the results of the study difficult to interpret (8). Further, adding NSAIDs and opioids to the LIA mixture is controversial and generally not recommended for elderly comorbid patients due to severe adverse effects such as kidney injuries and gastrointestinal disturbance (16).

The other study used LIA without NSAIDs (12). Their findings of reduced pain sensation, however, were after 36 and 60 hours postoperatively, which is quite late when reckoning that the LIA effect would last up to 24 hours (17). The study included only 12 patients in each group, which underlines the need for more confirmatory studies. Thus, our study provides knew knowledge indicating that LIA without NSAIDs might have an opioid-reducing effect in the first postoperative 24 hours after surgery for hemiarthroplasty patients.

The opioid-reducing effect of LIA in hip fracture patients is in contrast to studies on elective primary THA patients $(\mathbf{1 0 , 1 8 )}$. This may be explained by differences in the patient population such as old age, comorbidity, and low BMI, as the degree of ropivacaine-induced sensory and motor block is shown to be age and dose dependent (19). In addition, NSAIDs are not part of the multimodal pain regimen for hip fracture patients.

The effect of LIA with ropivacaine in hip fracture patients operated on with internal fixation has been investigated, finding no reduction in postoperative pain score or opioid consumption $(\mathbf{2 0 , 2 1 )}$. In these studies, LIA was given as a bolus dose intraoperatively, and thereafter postoperatively as repeated injections through a catheter placed intraoperatively for 48 hours. Also, these studies had only a few patients included, indicating that this might be a difficult patient population from which to recruit patients.

Studies investigating LIA in hip fracture patients in general report a low pain score in the early postoperative phase, demonstrating that the patients are well pain relieved $(\mathbf{1 1 , 1 2 )}$. In our study, the multimodal analgesic regimen included both preoperative dexamethasone and a femoral hip blockade. Dexamethasone is a long-acting prophylactic agent to reduce nausea and vomiting, and also to reduce postoperative pain. A single dose of dexamethasone provides postoperative pain relief (22) and is proven to be safe in hip fracture patients (23). The femoral hip blockade consisted of ropivacaine $2 \mathrm{mg} / \mathrm{mL}$ up to $40 \mathrm{~mL}$. Patients receiving the hip blockade less than 4 hours before surgery were excluded from the study. A singleshot nerve block is demonstrated to be effective as preopera- 
tive analgesia for hip fracture patients, reduces the needs for opioids before surgery and decreases postoperative delirium $(24,25)$. The use of preoperative femoral hip blockade alone has, however, not shown a reduction in postoperative opioid consumption (25).

The multimodal analgesic regimen and generally low pain scores might make it difficult to measure improved pain relief by using pain intensity as an outcome variable. It is therefore relevant also to consider secondary outcome variables such as opioid consumption, or level of activity. Our secondary outcome, opioid consumption on day 0 and on day 1 , was similar between the 2 study groups. However, when analyzing the results considering relevant covariates such as age, sex, and ASA group there was a difference in model estimate of $4.6 \mathrm{mg}$ opioid in favor of the LIA $(\mathrm{p}=0.04)$. This indicates some effect of adding intraoperative LIA with ropivacaine to the multimodal pain regimen following hemiarthroplasty. The difference of $4.6 \mathrm{mg}$ can be considered clinically relevant in respect of this elderly, fragile patient group.

\section{Limitations and strengths of the study}

Clinical studies on hip fracture patients with patient-reported outcomes might be challenging to execute, as the patients are elderly, fragile, and prone to postoperative delirium. The emergency admission and surgery are handled by on-call personnel who often have a busy working schedule. In this study, we reached the planned number of patients included, but pain scores postoperatively were collected for only roughly half of the patients. This is a major limitation of this study. There are several explanations for the missing data. First, the study was conducted in a university hospital with a major emergency department, involving different staff in the registration of pain scores. The work pressure is high in this setting and filling out a form to contribute to a clinical study might not be prioritized. Second, the study population are fragile with high comorbidity. Mobilization in the PACU was difficult and was abandoned for some patients. In addition, the transfer between the PACU and the orthopedic ward often happened late at night, and the patient was asleep or too tired to be mobilized or to set a pain score.

As some patients were operated on late at night, the opioid consumption on the day of surgery could vary among patients. An alternative method could have been to register data a given number of hours after surgery; however, the time of surgery was evenly distributed between the two study groups.

The strength of our study is the high number of patients included, compared with other studies on this patient population. In addition, data on postoperative opioid consumption was complete. Further, all patients were in a standardized orthogeriatric setting. All patients received a perioperative standardized multimodal pain regimen and were operated on under spinal anesthesia with the same surgical approach and the same surgical procedure. This makes the interpretation of the results valid.

\section{Conclusion}

In conclusion, local infiltration anesthesia with ropivacaine did not provide any reduction in pain score after fast-track hemiarthroplasty. Patients receiving ropivacaine, however, had a reduction in need for opioids.

All the authors contributed to planning of the study. JKH and TSW wrote the article and conducted the study. JKH, TSW, and JK conducted the data analyses and interpreted the results.

The authors thank the nurses and doctors who contributed to the registration of data for this study.

Acta thanks Henrik Husted for help with peer review of this study.

1. Casey S D, Stevenson D E, Mumma B E, Slee C, Wolinsky P R, Hirsch C H, et al. Emergency department pain management following implementation of a geriatric hip fracture program. West J Emerg Med 2017; 18: 585-91. doi: 10.5811/westjem.2017.3.32853

2. Gupta K, Nagappa M, Prasad A, Abrahamyan L, Wong J, Weingarten $\mathbf{T} \mathbf{N}$, et al. Risk factors for opioid-induced respiratory depression in surgical patients: a systematic review and meta-analyses. BMJ Open 2018; 8: e024086-e024086. doi: 10.1136/bmjopen-2018-024086

3. Morrison R S, Magaziner J, Gilbert M, Koval K J, McLaughlin M A, Orosz G, et al. Relationship between pain and opioid analgesics on the development of delirium following hip fracture. Journals Gerontology Ser A, Biol Sci Med Sci 2003; 58: 76-81. doi: 10.1093/gerona/58.1.m76

4. Yang Y, Zhao X, Dong T, Yang Z, Zhang Q, Zhang Y. Risk factors for postoperative delirium following hip fracture repair in elderly patients: a systematic review and meta-analysis. Aging Clin Exp Res 2017; 29: 11526. doi: 10.1007/s40520-016-0541-6

5. Cowan R, Lim J H, Ong T, Kumar A, Sahota O. The challenges of anaesthesia and pain relief in hip fracture care. Drugs Aging 2017; 34 : 1-11. doi: 10.1007/s40266-016-0427-5

6. Szatmári A, Menyhárt Á, Antal I, Sallai I, Holnapy G, Bejek Z, et al. The role of multimodal postoperative analgesia after primary hip arthroplasty. Orv Hetil 2019; 160: 2054-60. doi: 10.1556/650.2019.31556

7. Gelman D, Gelmanas A, Urbanaite D, Tamosiunas R, Sadauskas S, Bilskiene D, et al. Role of multimodal analgesia in the evolving enhanced recovery after surgery pathways. Medicina (Kaunas) 2018; 54. doi: 10.3390/medicina54020020

8. Kerr D R, Kohan L. Local infiltration analgesia: a technique for the control of acute postoperative pain following knee and hip surgery: a case study of 325 patients. Acta Orthop 2008; 79: 174-83. doi: $10.1080 / 17453670710014950$

9. Mont M A, Beaver W B, Dysart S H, Barrington J W, Del Gaizo D J. Local infiltration analgesia with liposomal bupivacaine improves pain scores and reduces opioid use after total knee arthroplasty: results of a randomized controlled trial. J Arthroplasty 2018; 33: 90-96. doi: 10.1016/j.arth.2017.07.024

10. Hofstad J K, Winther S B, Rian T, Foss O A, Husby O S, Wik T S. Perioperative local infiltration anesthesia with ropivacaine has no effect on postoperative pain after total hip arthroplasty. Acta Orthop 2015; 10.3109/17453674.2015.1053775: 1-5. doi: $10.3109 / 17453674.2015 .1053775$

11. Kang H, Ha Y C, Kim J Y, Woo Y C, Lee J S, Jang E C. Effectiveness of multimodal pain management after bipolar hemiarthroplasty for hip fracture: a randomized, controlled study. J Bone Joint Surg Am 2013; 95 : 2916. doi: 10.2106/jbjs.k.01708

12. Orapin J, Sutantavibul W, Chulsomlee K, Jarungvittayakon C, Pengrung N, Sirisreetreerux N, et al. Efficacy of periarticular multimodal drug injection without NSAIDs in elderly patients with displaced femoral neck fractures undergoing bipolar hemiarthroplasty: a prospective tripleblinded RCT. Cureus 2020; 12: e10271. doi: 10.7759/cureus.10271 
13. Prestmo A, Hagen G, Sletvold O, Helbostad J L, Thingstad P, Taraldsen K, et al. Comprehensive geriatric care for patients with hip fractures: a prospective, randomised, controlled trial. Lancet 2015; 385: 1623-3. doi: 10.1016/s0140-6736(14)62409-0

14. Caraceni A, Hanks G, Kaasa S, Bennett M I, Brunelli C, Cherny N, et al. Use of opioid analgesics in the treatment of cancer pain: evidencebased recommendations from the EAPC. Lancet Oncol 2012; 13: e58-68. doi: 10.1016/s1470-2045(12)70040-2

15. Fredheim O M, Husby K M, Kaasa S, Borchgrevink P C, Klepstad P. [Opioid switching and methadone in pain relief]. Tidsskr Nor Laegeforen 2006; 126: 2272-4

16. Fine M. Quantifying the impact of NSAID-associated adverse events. Am J Manag Care 2013; 19: s267-72.

17. Simpson D, Curran M P, Oldfield V, Keating G M. Ropivacaine: a review of its use in regional anaesthesia and acute pain management. Drugs 2005; 65: 2675-717. doi: 10.2165/00003495-200565180-00013

18. Lunn T H, Husted H, Solgaard S, Kristensen B B, Otte K S, Kjersgaard A G, et al. Intraoperative local infiltration analgesia for early analgesia after total hip arthroplasty: a randomized, double-blind, placebo-controlled trial. Reg Anesth Pain Med 2011; 36: 424-9. doi: 10.1097/AAP.0b013e3182186866

19. Simon M J, Veering B T, Stienstra R, van Kleef J W, Burm A G. The effects of age on neural blockade and hemodynamic changes after epidural anesthesia with ropivacaine. Anesth Analg 2002; 94: 1325-30, table of contents. doi: 10.1097/00000539-200205000-00052
20. Bech R D, Ovesen O, Lauritsen J, Emmeluth C, Lindholm P, Overgaard S. Local anesthetic wound infiltration after osteosynthesis of extracapsular hip fracture does not reduce pain or opioid requirements: a randomized, placebo-controlled, double-blind clinical trial in 49 patients. Pain Res Manage 2018; 2018: 6398424. doi: $10.1155 / 2018 / 6398424$

21. Bech R D, Lauritsen J, Ovesen O, Emmeluth C, Lindholm P, Overgaard S. Local anaesthetic wound infiltration after internal fixation of femoral neck fractures: a randomized, double-blind clinical trial in 33 patients. Hip Int 2011; 21: 251-9. doi: 10.5301/hip.2011.6513

22. De Oliveira G S, Jr., Almeida M D, Benzon H T, McCarthy R J. Perioperative single dose systemic dexamethasone for postoperative pain: a meta-analysis of randomized controlled trials. Anesthesiology 2011; 115: 575-88. doi: 10.1097/ALN.0b013e31822a24c2

23. Šakić L, Tonković D, Šakić K. Dexamethasone: intrathecal minimiser of simple haematologic stress biomarkers in hip fracture. Acta Clin Croat 2019; 58: 9-17. doi: 10.20471/acc.2019.58.s1.01

24. Li J, Dai F, Chang D, Harmon E, Ibe I, Sukumar N, et al. A practical analgesia approach to fragility hip fracture: a single-center, retrospective, cohort study on femoral nerve block. J Orthop Trauma 2019; 33: 175-9. doi: 10.1097/bot.0000000000001391

25. Steenberg J, Møller A M. Systematic review of the effects of fascia iliaca compartment block on hip fracture patients before operation. Br J Anaesth 2018; 120: 1368-80. doi: 10.1016/j.bja.2017.12.042 